\title{
Modules Over Color Hom-Poisson Algebras
}

\section{Ibrahima Bakayoko*}

Department de Mathematiques, UJNK/Centre Universitaire de N'Zerekore, BP: 50, N'Zerekore, Guinea

\begin{abstract}
In this paper we introduce color Hom-Poisson algebras and show that every color Hom-associative algebra has a non-commutative Hom-Poisson algebra structure in which the Hom-Poisson bracket is the commutator bracket. Then we show that color Poisson algebras (respectively morphism of color Poisson algebras) turn to color Hom-Poisson algebras (respectively morphism of Color Hom-Poisson algebras) by twisting the color Poisson structure. Next we prove that modules over color Hom-associative algebras A extend to modules over the color Hom-Lie algebras $L(A)$, where $L(A)$ is the color Hom-Lie algebra associated to the color Hom-associative algebra A. Moreover, by twisting a color Hom-Poisson module structure map by a color Hom-Poisson algebra endomorphism, we get another one.
\end{abstract}

Keywords: Color hom-associative algebras; Color hom-Lie algebras; Homomorphism; Formal deformation;Hom-modules; Modules over color Hom-Lie algebras; Modules over color Hom-Poisson algebras

\section{Introduction}

Color Hom-Poisson algebras are generalizations of Hom-Poisson algebras introducedin [1], where they emerged naturally in the study of 1-parameter formaldeformations of commutative Hom-associative algebras. Color Hom-Poisson algebrasgeneralize, on the one hand, color Hom-associative $[2,3]$ and color Hom-Lie algebras [2,3] which have been recently investigated by various authors. Onthe other hand, they generalize Hom-Lie superalgebras [4]. These structures arewell-known to physicists and to mathematicians studying differential geometry and homotopy theory. The cohomology theory of Lie superalgebras [5] has been generalizedto the cohomology of Hom-Lie superalgebras in [6]. A cohomology of colorLie algebras was introduced and investigated in [7], and the representations ofcolor Lie algebras were explicitly described in [8]. Modules over Poisson algebras receive various definitions $[9,10]$ we will use theone introduced in [9]. The aim of this paper is to study color Hom-Poisson algebras and modules over color Hom-Poisson algebras. The paper is organized as follows. In section 4 , we recall some basic notions related to color Hom-associative algebras and color Hom-Lie algebras. In section 5, we define color Hom-Poisson algebras and point out that to any color Hom-associative algebra ones can associate a color Hom-Poisson algebra. Next, starting from a color Poisson algebra and Poisson algebra morphism we get another one by twisting the associative product and Lie bracket. In section 6, we introduce modules over color Hom-Poisson algebras and prove that starting from a color HomPoisson module we get another one by twisting the module structure map by a Hom-Poisson algebra endomorphism. All vector spaces considered are supposed to be over fields of characteristics different from 2 .

\section{Preliminaries}

Let $\mathrm{G}$ be an abelian group. A vector space $\mathrm{V}$ is said to be a $\mathrm{G}$-graded if, there exist a family $\left(V_{a}\right)_{a} \in{ }_{\mathrm{G}}$ of vector subspaces of $\mathrm{V}$ such that

$$
V=\underset{a \in G}{\oplus} V_{a}
$$

An element $x \in V$ is said to be homogeneous of degree $a \in G$ if $x \in$ $V_{a}$ We denote $\mathrm{H}(\mathrm{V})$ the set of all homogeneous elements in $\mathrm{V}$.

Let $V=\underset{a \in G}{\oplus} V_{a}$ and $V^{\prime}=\oplus_{a \in G} V_{a}^{\prime}$ be two G-graded vector spaces. A linear mapping $f: V \rightarrow V^{\prime}$ is said to be homogeneous of degree $\mathrm{b}$ if $f\left(V_{a}\right) \subseteq V_{a+b}, \forall_{a} \in G$
If, fis homogeneous of degree zero i.e. $f\left(V_{a}\right) \subseteq V_{a}^{\prime}$ holds for any $a \in$ $G$ then $f$ is said to be even.

An algebra $(A, \mu)$ is said to be G-graded if its underlying vector space is G-graded i.e. $A=\oplus_{a \in G} A_{a}$ and if furthermore $\mu\left(A_{a}, A_{b}\right) \subseteq A_{a+b}$ for all $a, b \in \mathrm{G}$.

Let $A^{\prime}$ be another G-graded algebra. A morphism $f: A \rightarrow A^{\prime}$ of G-graded algebras is by definition an algebra morphism from $A$ to $A^{\prime}$ which is, in addition an even mapping.

\section{Definition}

Let $\mathrm{G}$ be an abelian group. A map $\varepsilon: G \mathrm{XX} \rightarrow K^{*}$ is called a skewsymmetric bicharacter on $\mathrm{G}$ if the following identities hold,

$$
\begin{aligned}
& \text { 1. } \varepsilon(\mathrm{a}, \mathrm{b}) \varepsilon(\mathrm{b}, \mathrm{a})=1 \\
& \text { 2. } \varepsilon(\mathrm{a}, \mathrm{b}+\mathrm{c})=\varepsilon(\mathrm{a}, \mathrm{b}) \varepsilon(\mathrm{a}, \mathrm{c}) \\
& \text { 3. } \varepsilon(\mathrm{a}+\mathrm{b}, \mathrm{c})=\varepsilon(\mathrm{a}, \mathrm{c}) \varepsilon(\mathrm{b}, \mathrm{c}) \\
& \mathrm{a}, \mathrm{b}, \mathrm{c} \in \mathrm{G}
\end{aligned}
$$

Remark that

$$
\varepsilon(a, 0)=\varepsilon(0, a)=1, \varepsilon(a, a)= \pm 1 \text { For all } a \in G
$$

Where, 0 is the identity of $\mathrm{G}$. If $\mathrm{x}$ and $\mathrm{y}$ are two homogeneous elements of degree a and $\mathrm{b}$ respectively and $\varepsilon$ is a skew-symmetric bicharacter, then we shorten the notation by writing $\varepsilon(x, y)$ instead of $\varepsilon(a, b)$

\section{Definition}

A color Hom-associative algebra is a quadruple $(A, \mu, \varepsilon, \alpha)$ consisting of a G-graded vector space $\mathrm{A}$, an even bilinear map $\mu: A \mathrm{X} A \rightarrow A$ and an even linear map such $\alpha: A \rightarrow A$ that

*Corresponding author: Ibrahima Bakayoko, Department de Mathematiques, UJNK/Centre Universitaire de N'Zerekore, BP: 50, N'Zerekore, Guinea, E-mail: ibrahimabakayoko27@gmail.com

Received August 12, 2013; Accepted September 30, 2014; Published October 06, 2014

Citation: Bakayoko I (2014) Modules Over Color Hom-Poisson Algebras. J Generalized Lie Theory Appl 8: 212. doi: 10.4172/1736-4337.1000212

Copyright: (c) 2014 Bakayoko I. This is an open-access article distributed under the terms of the Creative Commons Attribution License, which permits unrestricted use, distribution, and reproduction in any medium, provided the original author and source are credited. 


$$
\mu(\alpha(x), \alpha(y))=\alpha(\mu(x, y))
$$

$$
\mu(\alpha(x), \mu(y, z))=\mu(\mu(x, y), \alpha(z))
$$

If in addition $\mu(x, y)=\varepsilon(x, y) \mu(y, x)$ the color Hom-associative algebra $(A, \mu, \varepsilon, \alpha)$ is said to be a $\varepsilon$-commutative color Hom-associative algebra.

\section{Remark}

When $\alpha=I d$ we recover the classical associative color algebra.

Recall that the Hom-associator, $a S_{A}$ of a Hom-algebra A is defined as : as $A: A \otimes A \otimes A \rightarrow A$,

Observe that $a s_{A} \equiv 0$ when A is a color-Hom-associative algebra.

\section{Definition}

Let $(A, \mu, \varepsilon, \alpha)$ and $\left(A^{\prime}, \mu^{\prime}, \varepsilon^{\prime}, \alpha^{\prime}\right)$ be two color Hom-associative algebras. An even linear map $f: A \rightarrow A^{\prime}$ is said to be a morphism of color Hom-associative algebras if $f \circ \alpha=\alpha^{\prime} \circ f$ and

$$
f(\mu(x, y))=\mu^{\prime}(f(x), f(y))
$$

For all $x, y \in A$.

\section{Lemma}

([17]) Let $(A, \mu, \varepsilon$,$) be a color associative algebra and \alpha$ be an even algebra endomorphism. Then $(A, \mu \alpha, \varepsilon, \alpha)$ where $\mu_{\alpha}=\alpha \circ \mu$ is a color Hom-associative algebra. Moreover, suppose that $\left(A^{\prime}, \mu^{\prime}, \varepsilon\right)$ be another color associative algebra and $\alpha^{\prime}: A^{\prime} \rightarrow A^{\prime}$ be an even algebra endomorphism such that $f \circ \alpha=\alpha^{\prime} \circ f$ then $f:\left(A, \mu^{\prime}, \varepsilon, \alpha\right) \rightarrow\left(A^{\prime}, \mu^{\prime}, \varepsilon, \alpha^{\prime}\right)$ is also a morphism of color Hom-associative algebras.

\section{Definition}

([17]) A color Hom-Lie algebra is a quadruple $(A,\{. .\},, \varepsilon, \alpha)$ consisting of a G-graded vector space $\mathrm{A}$, an even bilinear map

$\{.,\}:. A X A \rightarrow A$ (i.e $\left\{A_{a}, A_{b}\right\} \subseteq A_{a+b}$ for all $a, b \in G$ ) a bicharacter, and an even linear map $\alpha: A \rightarrow A$ such that for any $x, y \in H(A)$ we have

$$
\begin{array}{ll}
\{x, y\}=-\varepsilon(x, y)\{y, x\} & (\varepsilon \text {-Skew-symmetry) } \\
\alpha \circ\left\{, .,=\{.,\} \circ \alpha^{\otimes 2}\right. & \text { (Multiplicativity) } \\
\oint \varepsilon(z, x)\{\alpha(x),\{y, z\}\}=0 & (\varepsilon \text {-Hom-Jacobi identity) }
\end{array}
$$

Where $\oint$ means cyclic summation.

By the $\varepsilon$ skew symmetry 3 of the color Hom-Lie bracket $\{.$, . $\}$, the color Hom-Jacobi identity 5 is equivalent to

$$
\{\{x, y\}, \alpha(z)\}=\{\alpha(x),\{y, z\}\}-\varepsilon(x, y)\{\alpha(y),\{x, z\}\}
$$

Remark that a color Lie algebra $(A,\{.,\},. \varepsilon)$ is a color Hom-Lie algebra with $\alpha=$ Id

Morphism of color Hom-Lie algebras are defined similarly to the Definition 4.3, where the color Hom-associative product is replaced by the color Hom-Lie bracket. Examples of color Hom-Lie algebras are provided in $[2,3]$.

The following lemma connects color Hom-associative algebras to color Hom-Lie algebras.

\section{Lemma}

([17]) Let $(A, \mu, \varepsilon, \alpha)$ be a color Hom-associative algebra.
Then $\left(A,\{.\}=.\mu-\varepsilon(..) \mu^{o p}, \varepsilon, \alpha\right)$ is a color Hom-Lie algebra, denoted by $\mathrm{L}(\mathrm{A})$.

\section{Color Hom-Poisson algebras}

\section{Definition}

A color Hom-Poisson algebra consists of a G-graded vector space A, a multiplication $\mu: A \mathrm{X} A \rightarrow A$, an even bilinear bracket $\{.\}:. A \mathrm{X} A \rightarrow A$ and an even linear map $\alpha: A \rightarrow A$ such that

1. $(A, \mu, \varepsilon, \alpha)$ is a color Hom-associative algebra,

2. $(A,\{. .\},, \varepsilon, \alpha)$ is a color Hom-Lie algebra,

3. the color Hom-Leibniz identity is satisfied i.e.

$\{\alpha(x), \mu(y, z)\}=\mu(\{x, y\}, \alpha(z))+\varepsilon(x, y) \mu(\alpha(y),\{x, z\})$,

For $x, y, z \in H(A)$ any

If in addition $\mu$ is $\varepsilon$ commutative, the color Hom-Poisson algebra $(A,\{.,\},, \varepsilon, \alpha)$ is said to be a $\varepsilon$ commutative color Hom-Poisson algebra.

The condition 7 expresses the compatibility between the color Hom-associative product $\mu$ and the color Hom-Lie bracket $\{. .$,$\} it can$ be written equivalently

$$
\{\mu(x, y), \alpha(z)\}=\mu(\alpha(x),\{y, z\})+\varepsilon(x, y) \mu(\alpha(y),\{x, z\}) .
$$

\section{Remark}

We recover Poisson algebras $([6,5])$ when $\alpha=\operatorname{Id}$ and $\varepsilon \equiv 1$

We need the following lemma in Proposition 6.1.

\section{Lemma}

If $(A, \mu\{. .\},, \varepsilon, \alpha)$ is a $\varepsilon$ commutative color Hom-Poisson algebra then $(A,-\mu-\{.,\},. \varepsilon, \alpha)$ is also a $\varepsilon$ commutative color Hom-Poisson algebra. 4.6).

The following theorem is the color version of ([11], Proposition

\section{Theorem}

Let $(A, \mu, \varepsilon, \alpha)$ be a color Hom-associative algebra.

Then $\left(A, \mu,\{.,\}=.\mu-\varepsilon(.,.) \mu^{o p}, \varepsilon, \alpha\right) \sqrt{b^{2}-4 a c}$ is a color Hom-Poisson algebra.

Proof:

According to Lemma 4.2, it remains to prove the color HomLeibniz identity 7 . For any $x, y, z \in H(A)$

$$
\begin{aligned}
& \{\alpha(x), \mu(y, z)\}-\mu(\{x, y\}, \alpha(z))-\varepsilon(x, y) \mu(\alpha(y),\{x, z\})= \\
& =\mu(\alpha(x), \mu(y, z))-\varepsilon(x, y=z) \mu(\mu(x, y), \alpha(z)) \\
& =\varepsilon(x,) \mu(\mu(y, x), \alpha(z))-\varepsilon(x, y) \mu(\alpha(y), \mu(x, z))+\varepsilon(x, y) \varepsilon(x, z) \mu(\alpha(y), \mu(z, x)) \\
& =\mu(\alpha(x), \mu(y, z))-\mu(\mu(x, y), \alpha(z))-\varepsilon(x, y+z) \mu(\mu(y, z), \alpha(x)) \\
& +\varepsilon(x, y) \mu(\mu(y, x), \alpha(z))+\varepsilon(x, y) \mu(\mu(y, x), \alpha(z))-\varepsilon(x, y) \mu(\alpha(y), \mu(, z)) \\
& =-a s_{\mu, \alpha}(x, y, z)-\varepsilon(x,) \varepsilon(x, z) a s_{\mu, \alpha}(y, z, x)+\varepsilon(x, y) a s_{\mu, \alpha}(y, x, z)=0
\end{aligned}
$$

This finishes the proof.

\section{Corollary}

Let $(A, \mu\{. .\},, \varepsilon, \alpha)$ be a color associative algebra and $\alpha$ an even color algebra endomorphism. Then $\left(A, \mu_{\alpha},\{\ldots,\}_{\alpha}, \varepsilon, \alpha\right)$ where 
$\mu_{\alpha}=\alpha \circ \mu,\{.,\}_{\alpha}=\mu_{\alpha}-\varepsilon(.,.) \mu_{\alpha}^{o p}$ is a color Hom-Poisson algebra.

\section{Proof}

The proof follows from Lemma 4.1 and Theorem 3.1.

\section{Lemma}

Let $(A, \mu\{.,\},. \varepsilon)$ be a color Poisson algebra and $\alpha$ be an even color Poisson algebra endomorphism. Then $\left(A, \mu_{\alpha},\{.,\}_{\alpha}, \varepsilon, \alpha\right)$ is a color Hom Poisson algebra.

\section{Proof}

By Lemma 4.1 and ([3] Example1.2), we only need to prove the color Hom-Leibniz identity. For any $x, y, z \in H(A)$

$$
\begin{aligned}
& \left\{\alpha(x), \mu_{\alpha}(y, z)\right\}_{\alpha}-\mu_{\alpha}\left(\{x, y\}_{\alpha} \alpha(z)\right)-\varepsilon(x, y) \mu_{\alpha}\left(\alpha(y),\{x, z\}_{\alpha}\right)= \\
& \left.=\alpha^{2}(x), \alpha^{2}(\mu(y, z))\right\}-\mu\left(\alpha^{2}(\{x, y\}), \alpha^{2}(z)\right)-\varepsilon(x, y) \mu\left(\alpha^{2}(y), \alpha^{2}(\{\mathrm{x}, z\})\right) \\
& =\alpha^{2}(\{x, \mu(y, z)\}-\mu(\{x, y\}, z)-\varepsilon(x, y) \mu(y,\{x, z\})) \\
& =0
\end{aligned}
$$

This completes the proof.

\section{Theorem}

Let $(A, \mu\{. .\},, \varepsilon, \alpha)$ be a color Hom-Poisson algebra and $\beta: A \rightarrow A$ be an even color Poisson algebra endomorphism. Then, $A_{\beta}=\left(A, \mu_{\beta}=\beta \circ \mu,\{.,\}_{\beta}=\beta \circ\{.,\},. \varepsilon, \beta \circ \alpha\right)$ is a color Hom-Poisson algebra.

Moreover, suppose that $\left(A^{\prime}, \mu^{\prime},\{. .\}^{\prime} \varepsilon\right)$ is a color Poisson algebra and $\left(A,\{. .,\}_{\beta}, \varepsilon, \beta \circ \alpha\right)$ is an even color Poisson algebra endomorphism. If $f: A \rightarrow A^{\prime}$ is a color Poisson algebra morphism that satisfies $f \circ \beta=\alpha^{\prime} \circ f$ , then

$f:\left(A, \mu_{\beta},\{., .\}_{\beta}, \varepsilon, \beta \circ \alpha\right) \rightarrow\left(A^{\prime}, \mu_{\alpha}^{\prime},\{.,\}_{\alpha}^{\prime}, \varepsilon, \alpha\right)$

is a color Hom-Poisson algebra homomorphism.

\section{Proof}

It is straightforward to show that $\left(A, \mu_{\beta}, \varepsilon, \beta \circ \alpha\right)$ is a color Hom associative algebra and $\left(A,\{.,\}_{\beta}, \varepsilon, \beta \circ \alpha\right)$ is a color Hom-Lie algebra ([3] Theorem1.1). The proof of the color Hom-Leibniz identity is similar to that of Lemma 5.2.For the second assertion, we have

$$
\begin{aligned}
& f\left(\mu_{\beta}(x, y)\right)=f(\mu(\beta(x), \beta(y)))=\mu^{\prime}(f(\beta(x)), f(\beta(y))) \\
& =\mu^{\prime}\left(\alpha^{\prime}(f(x)), \alpha^{\prime}(f(y))=\mu_{\alpha^{\prime}}^{\prime}(f(x),(y)) .\right.
\end{aligned}
$$

We have a similar proof for the color Hom-Poisson bracket.

\section{Corollary 5.2}

Let $(A, \mu\{.,\},. \varepsilon, \alpha)$ be a color Hom-Poisson algebra. Then

$$
A^{n}=\left(A,\{., .\}^{(n)}=\alpha^{n} \circ\{., .\}, \mu^{(n)}=\alpha^{n} \circ \mu, \varepsilon, \alpha^{n+1}\right)
$$

is a color Hom-Poisson algebra for each integer $\mathrm{n} \geq 0$. We finish this section by studying deformations by composition of color HomPoisson algebras.

\section{Definition 5.2}

Let $(A, \mu\{. .\},, \varepsilon, \alpha$ )be a color Hom-Poisson algebra. A one parameter formal deformation of $\mathrm{A}$ is given by $\mathrm{K}[\mathrm{t}]]$-bilinear maps $\mu_{t}(.):. A[[t]] \mathrm{X} A[[t]] \rightarrow A[[t]]$ and $\{. .,\}_{t}: A[[t]] \mathrm{X} A[[t]] \rightarrow A[[t]]$ of the form $\mu_{t}=\sum_{i>0} t^{i} \mu_{i}$ and $\{.,\}=.\sum_{i>0} t^{i}\{.,\}_{i}$ where each $\mu_{i}$ and $\{.,\}_{i}$ are K-bilinear maps and (extended to K[[t]]-bilinear maps), and such that for all following conditions be satisfied.

$$
\begin{aligned}
& \mu_{t}\left(\alpha_{t}(x), \alpha_{t}(y)\right)=\alpha_{\mathrm{t}}\left(\mu_{t}(x, y)\right), \\
& \mu_{t}\left(\alpha_{t}(x), \mu_{t}(y, z)\right)=\mu_{t}\left(\mu_{t}(x, y), \alpha_{t}(z)\right), \\
& \alpha_{t} \circ\{., .\}_{t}=\{., .\}_{t} \circ \alpha_{t}^{\otimes 2}, \\
& \{x, y\}_{t}=-\varepsilon(x, y)\{y, x\}_{t}, \\
& \oint \varepsilon(z, x)\left\{\alpha_{t}(x),\{y, z\}_{t}\right\}_{t}=0, \\
& \left\{\alpha_{t}(x), \mu_{t}(y, z)\right\}_{t}=\mu_{t}\left(\{x, y\}_{t}, \alpha_{t}(z)\right)+\varepsilon(x, y) \mu_{t}\left(\alpha_{t}(y),\{x, z\}_{t}\right) .
\end{aligned}
$$

The deformation is said to be of order $\mathrm{k}$ if $\mu_{t}=\sum_{i=0}^{k} t^{i} \mu_{i}$ and $\{., .\}_{t}=\sum_{i=0}^{k} t^{i}\{., .\}_{i}$

\section{Proposition 5.1}

Let $(A, \mu\{.,\},. \varepsilon)$ be a color Poisson algebra and $\alpha_{t}$ an even color Poisson algebra endomorphism of the form $\alpha_{t}=\alpha_{0}+\sum_{i=0}^{k} t^{i} \alpha_{i}$ where $\alpha_{i}$ are endomorphism of $\mathrm{A}$ (as color Poisson algebra), $\mathrm{t}$ is a parameter in $\mathrm{K}$ and $\mathrm{k}$ is an entiger. Let $\mu_{t} \quad \alpha_{t} \mu$ and $\{., .\}_{t}=\alpha_{t} \circ\{.,$. then $\left(A, \mu_{t},\{., .\}_{t}, \varepsilon, \alpha_{t}\right)$ is a color Hom-Poisson algebra which is a deformation of the color Poisson algebra $(A, \mu\{.,\},. \varepsilon$ viewed as a color Hom-Poisson algebra $(A, \mu\{. .\},. \mathcal{E}, I d)$.

\section{Proof}

The proof follows from Theorem.2.

As in the case of Poisson algebras $([10,12,13])$, the cohomology of color Hom-Poisson algebras is described by the cohomology of the underlying color Hom-Lie algebras ([3]).

\section{Modules Over Color Hom-Poisson Algebras}

\section{Definition 6.1}

Let $\mathrm{G}$ be an abelian group. A Hom-module is a pair $\left(M, \alpha_{M}\right)$ in which $\mathrm{M}$ is a G-graded vector space and $\alpha_{M}: M \rightarrow M$ is an even linear map.

\section{Definition 6.2}

Let $\left(A, M_{A}, \varepsilon, \alpha_{A}\right)$ be a color Hom-associative algebra. An Amodule is a Hom-module $\left(M, \alpha_{M}\right)$ together with a bilinear map $\mu_{M}: A \otimes M \rightarrow M$ called structure map, such that

$$
\begin{aligned}
& \mu_{M}\left(A_{a} \cdot M_{b}\right) \subseteq M_{a+b} \\
& \alpha_{M} \circ \mu_{M}=\mu_{M} \circ\left(\alpha_{A} \otimes \alpha_{M}\right), \\
& \mu_{M} \circ\left(\alpha_{A} \otimes \mu_{M}\right)=\mu_{M} \circ\left(\mu_{A} \otimes \alpha_{M}\right)
\end{aligned}
$$

Twisting a module structure map by an algebra endomorphism, we get another one as stated in the following Lemma.

\section{Lemma 6.1}

Let $\left(A, M_{A}, \varepsilon, \alpha_{A}\right)$ be a color Hom-associative algebra and $\mathrm{M}$ an A-module with structure map $\mu_{M}: A \otimes M \rightarrow M$ Define the map

$$
\tilde{\mu}_{M}=\mu_{M} \circ\left(\alpha_{A}^{2} \otimes I d_{M}\right): A \otimes M \rightarrow M .
$$

Then $\mu_{M}$ is the structure map of another A-module structure on M. 


\section{Proof}

The proof is similar to that of ([14], Lemma 4.5).

\section{Definition 6.3}

([3]) Let $\left(L,\{.,\},. \varepsilon, \alpha_{L}\right)$ be a color Hom-Lie algebra and $\left(M, \alpha_{M}\right)$ a Hom-module. An L-module on $\mathrm{M}$ consists of a K-bilinear map $\mu_{M}: A \otimes M \rightarrow M$ such that

$$
\begin{aligned}
& \mu_{M}\left(A_{a}, M_{b}\right) \subseteq M_{a+b} \\
& \alpha_{M}\left(\mu_{M}(\mathrm{x}, \mathrm{m})\right)=\mu_{\mathrm{M}}\left(\alpha_{L}(\mathrm{x}), \alpha_{\mathrm{M}}(m)\right), \\
& \mu_{M}\left(\{\mathrm{x}, \mathrm{y}\}, \alpha_{M}(\mathrm{~m})\right)=\mu_{\mathrm{M}}\left(\alpha_{L}(\mathrm{x}), \mu_{M}(, m)\right) \\
& -\varepsilon(x, y) \mu_{M}\left(\alpha_{L}(\mathrm{y}), \mu_{\mathrm{M}}(\mathrm{x}, \mathrm{m})\right),
\end{aligned}
$$

for any $\mathrm{m} \in H(\mathrm{M}), \mathrm{x}, \mathrm{y} \in H(L)$

\section{Remark 6.1}

When $\alpha_{M}=I d_{M}$ and $\alpha=I d_{L}$ we recover the definition of Lie modules ([15-17]).

The following statement is the Lie analogue of Lemma 6.1.

\section{Lemma 6.2}

Let $(L,\{. .\},, \mathcal{E}, \alpha)$ be a color Hom-Lie algebra and $\mathrm{M}$ an L-module with structure map $\mu_{M}=L \otimes M \rightarrow M$ Define the map

$$
\tilde{\mu}_{M}=\mu_{M} \circ\left(\alpha^{2} \otimes I d_{M}\right): L \otimes M \rightarrow M
$$
M.

Then $\mu_{M}$ is the structure map of another L-module structure on

\section{Proof}

Equalities 19 and 20 are proved as in Lemma 6.1. Now, we prove

21 for $\mu_{M}$. For any $x, y \in L, m \in M$

$$
\begin{aligned}
\tilde{\mu}_{M}\left(\{x, y\}, \alpha_{\mathrm{M}}(\mathrm{m})\right)= & \mu_{M}\left(\alpha^{2} \otimes I d\right)\left(\{x, y\}, \alpha_{M}(m)\right) \\
= & \mu_{M}\left(\left\{\alpha^{2}(x), \alpha^{2}(y)\right\}, \alpha_{M}(m)\right) \\
= & \mu_{M}\left(\alpha^{3}(\mathrm{x}), \mu_{\mathrm{M}}\left(\alpha^{2}(\mathrm{y}), \mathrm{m}\right)\right)-\varepsilon(\mathrm{x}, \mathrm{y}) \mu_{\mathrm{M}}\left(\alpha^{3}(\mathrm{y}), \mu_{\mathrm{M}}\left(\alpha^{2}(x), m\right)\right) \\
= & \mu_{M}\left(\alpha^{3}(x), \mu_{M}\left(\alpha^{2} \otimes I d\right)(\mathrm{y} \otimes \mathrm{m})\right) \\
& -\varepsilon(\mathrm{x}, \mathrm{y}) \mu_{\mathrm{M}}\left(\alpha^{3}(y), \mu_{M}\left(\alpha^{2} \otimes I d\right)(x \otimes m)\right) \\
= & \mu_{M}\left(\alpha^{2}\left(\alpha(x), \tilde{\mu}_{M}(y \otimes m)\right)-\varepsilon(x, y) \mu_{M}\left(\alpha^{2}(\alpha(Y)), \tilde{\mu}_{M}(\mathrm{x} \otimes \mathrm{m})\right)\right. \\
= & \mu_{M}\left(\alpha^{2} \otimes I d\right)\left(\alpha(x) \otimes \tilde{\mu}_{M}(y \otimes m)\right) \\
& -\varepsilon(x, y) \mu_{M}\left(\alpha^{2} \otimes I d\right)\left(\alpha(\mathrm{y}) \otimes \tilde{\mu}_{M}(x \otimes m)\right) \\
= & \tilde{\mu}_{M}\left(\alpha(\mathrm{x}), \tilde{\mu}_{M}(y, m)\right)-\varepsilon(x, y) \tilde{\mu}_{M}\left(\alpha(y), \tilde{\mu}_{M}(x, m)\right) .
\end{aligned}
$$

Hence the conclusion holds.

The following result shows that A-modules extend to $\mathrm{L}(\mathrm{A})$-modules with samemodule structure map.

\section{Theorem 6.1}

Let $(A, \mu, \varepsilon, \alpha)$ be a color Hom-associative algebra and $\left(M, A_{M}\right)$ an A-module with structure map $\mu_{M}$. Then, $\mathrm{M}$ is a $\mathrm{L}(\mathrm{A})$-module with structure map $\mu_{M}$.

\section{Proof}

In fact, it suffices to show the relation 21. For any $x, y \in H(A), m \in H(M)$, We have

$$
\begin{aligned}
& \mu_{M}\left(\alpha(x), \mu_{M}(y, m)\right)-\varepsilon(x, y) \mu_{M}\left(\alpha(y), \mu_{M}(x, m)\right) \\
= & \mu_{M}\left(\mu(x, y), \alpha_{M}(m)\right)-\varepsilon(x, y) \mu_{M}\left(\mu(y, x), \alpha_{M}(m)\right) \\
= & \mu_{M}\left(\mu(x, y)-\varepsilon(x, y) \mu(y, x), \alpha_{M}(m)\right) \\
= & \mu_{M}\left(\{x, y\}, \alpha_{M}(\mathrm{~m})\right) .
\end{aligned}
$$

This establishes the Theorem.

The corollaries below give a large class of examples of $\mathrm{L}(\mathrm{A})$ modules.

\section{Corollary 6.1}

Let $A_{\alpha}=\left(\mathrm{A}, \mu_{\alpha}, \varepsilon, \alpha\right)$ be a color Hom-associative algebra as in Lemma 4.1 and $\left(M, \alpha_{M}\right)$ an $A_{\alpha}$ module with structure map $\mu_{M}$ Then, $\mathrm{M}$ is an $\mathrm{L}(\mathrm{A})$-module with structure map $\mu_{M}$.

Proof

Prove 21. Indeed, for $x, y \in H(A), m \in H(M)$ we have

$$
\begin{aligned}
& \mu_{M}\left(\alpha(x), \mu_{M}(y, m)\right)-\varepsilon(x, y) \mu_{M}\left(\alpha(y), \mu_{M}(x, m)\right) \\
= & \left.\mu_{M}\left(\mu_{\alpha}(x, y)\right), \alpha_{M}(m)\right)-\varepsilon(x, y) \mu_{M}\left(\mu_{\alpha}(y, x), \alpha_{M}(m)\right) \\
= & \mu_{M}\left(\mu_{\alpha}(x, y)-\varepsilon(x, y) \mu_{\alpha}(y, x), \alpha_{M}(m)\right) \\
= & \mu_{M}\left(\{x, y\}, \alpha_{M}(\mathrm{~m})\right) .
\end{aligned}
$$

We conclude that $\mathrm{M}$ is an $\mathrm{L}(\mathrm{A})$-module with structure map $\mu_{M}$

\section{Corollary 6.2}

Let $(A, \mu, \varepsilon, \alpha)$ be a color Hom-associative algebra and $\left(M, \alpha_{M}\right)$ an A-module with structure map $\mu_{M}$ Put

$\tilde{\mu}_{M}=\mu_{M} \circ\left(\alpha_{A}^{2} \otimes I d_{M}\right)$

Then $\mathrm{M}$ is an $\mathrm{L}(\mathrm{A})$-module with structure map $\tilde{\mu}_{M}$.

Proof

We know from Lemma (6.1) that $\tilde{\mu}_{M}$ is an A-module structure map. And, for $x, y \in H(A), m \in H(M)$, one has

$$
\begin{aligned}
& \tilde{\mu}_{M}\left(\alpha(x), \tilde{\mu}_{M}(y, m)\right)-\varepsilon(x, y) \tilde{\mu}_{M}\left(\alpha(\mathrm{y}), \tilde{\mu}_{M}(\mathrm{x}, m)\right) \\
= & \mu_{M}\left(\alpha^{2}(\alpha(x)), \mu_{M}\left(\alpha^{2}(y), m\right)\right)-\varepsilon(x, y) \mu_{M}\left(\alpha^{2}(\alpha(\mathrm{y})), \mu_{M}\left(\alpha^{2}(\mathrm{x}), m\right)\right) \\
= & \mu_{M}\left(\alpha\left(\alpha^{2}(x)\right), \mu_{M}\left(\alpha^{2}(y), m\right)\right)-\varepsilon(x, y) \mu_{M}\left(\alpha\left(\alpha^{2}(\mathrm{y})\right), \mu_{M}\left(\alpha^{2}(\mathrm{x}), m\right)\right) \\
= & \mu_{M}\left(\mu\left(\alpha^{2}(x), \alpha^{2}(y)\right), \alpha_{M}(m)\right)-\varepsilon(x, y) \mu_{M}\left(\mu\left(\alpha^{2}(\mathrm{y}), \alpha^{2}(\mathrm{x})\right), \alpha_{M}(m)\right) \\
= & \mu_{M}\left(\alpha^{2}(\mu(x, y)-\varepsilon(x, y) \mu(y, x)), \alpha_{M}(m)\right) \\
= & \mu_{M}\left(\alpha^{2}(\{x, y\}), \alpha_{M}(m)\right) \\
= & \tilde{\mu}_{M}\left(\{x, y\}, \alpha_{M}(m)\right) .
\end{aligned}
$$

This is similar to the relation 21 for $\tilde{\mu}_{M}$

Now we define modules for color Hom-Poisson algebras.

\section{Definition 6.4}

Let $(A, \mu,\{. .\},, \varepsilon, \alpha)$ be a $\varepsilon$ commutative color Hom-Poisson algebra and $\left(M, \alpha_{M}\right)$ a Hom-module. 
A color Hom-Poisson module structure on $\mathrm{M}$ consists of two K-bilinear maps $\mu_{M}: A \otimes M \rightarrow M$ and $\lambda_{M}: A \otimes M \rightarrow M$ such that

(i) $\mathrm{M}$ is an A-module and an L-module,

(ii) And for any $x, y \in H(A), m \in H(M)$,

$$
\begin{aligned}
\lambda_{M}\left(\alpha(x), \mu_{M}(y, m)\right)= & \mu_{M}\left(\{x, y\}, \alpha_{M}(m)\right) \\
& +\varepsilon(x, y) \mu_{M}\left(\alpha(y), \lambda_{M}(x, m)\right), \\
\lambda_{M}\left(\mu(x, y), \alpha_{M}(m)\right)= & \mu_{M}\left(\alpha(x), \lambda_{M}(y, \mathrm{~m})\right. \\
& +\varepsilon(x, y) \mu_{M}\left(\alpha(y), \lambda_{M}(x, m)\right) .
\end{aligned}
$$

When $\alpha=I d_{A}, \alpha_{M}=I d_{M}$ and $\varepsilon \equiv 1$

We recover the definition of modules over Poisson algebras ([9]).

\section{Example 6.1}

(i) Any module over a $\varepsilon$ commutative color Hom-associative algebra (resp. color Hom-Lie algebra) can be seen as a module over a $\varepsilon$ commutative color Hom-Poisson algebra with the trivial color HomLie bracket (resp. trivial color Hom-associative product).

(ii) Any $\varepsilon$ commutative color Hom-Poisson algebra is a module over itself

\section{Example 6.2}

Let $\left(V, \mu_{v}, \lambda_{v}, \alpha_{V}\right)$ and $\left(W, \mu_{W}, \lambda_{W}, \alpha_{W}\right)$ be two modules over the $\varepsilon$ Commutative color Hom-Poisson algebra $(A, \mu,\{. .\},, \varepsilon, \alpha)$

Then the direct product $M=V \times W$ is a module over A with structure maps $\mu_{M}: A \otimes M \rightarrow M, \lambda_{M}: A \otimes M \rightarrow M$ and $\alpha_{M}: M \rightarrow M$

\section{Defined by}

$\mu_{M}(x(v, w))=\left(\mu_{V}(x, v), \mu_{W}(x, \mathrm{w})\right), \lambda_{M}(x,(\mathrm{v}, \mathrm{w}))=\left(\lambda_{Y}(x, v), \lambda_{W}(x, \mathrm{w})\right)$ and $\alpha_{w}(v, w)=\left(\alpha_{V}(v), \alpha_{W}(w)\right)$ for any $x \in H(A), v \in H(V)$ and $w \in H(W)$

\section{Proposition 6.1}

If $\left(M, \mu_{M}, \lambda_{M} \alpha_{M p}\right)$ is a module over the $\varepsilon$ commutative color HomPoisson algebra $(A, \mu,\{. .\},. \varepsilon, \alpha)$ then $\left(M,-\mu_{M^{\prime}}-\lambda_{M^{\prime}} \alpha_{M^{\prime}}\right)$ is also a module $\varepsilon, \alpha)$

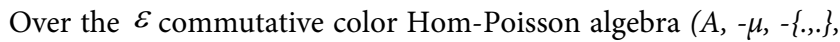

\section{Proof}

The proof comes from Definition 6.4 and Lemma 5.1.

\section{Theorem 6.2}

Let $(A, \mu,\{. .\},. \varepsilon, \alpha)$ be A $\varepsilon$ commutative color Hom-Poisson algebra and $\left(M, \mu_{M^{\prime}} \lambda_{M^{\prime}} \alpha_{M^{\prime}}\right)$ color Hom-Poisson module. Then

$$
\begin{aligned}
& \tilde{\mu}_{M}=\mu_{M} \circ\left(\alpha^{2} \otimes I d_{M}\right): \mathrm{A} \otimes M \rightarrow M, \\
& \tilde{\lambda}_{M}=\lambda_{M} \circ\left(\alpha^{2} \otimes I d_{M}\right): \mathrm{A} \otimes M \rightarrow M,
\end{aligned}
$$

Define another color Hom-Poisson module structure on M.

\section{Proof}

We know that $\mu_{M}$ is a structure of another A-module structure map on M (Lemma 6.1) and $\tilde{\lambda}_{M}$ is a structure of another L-module structure map on M (Lemma 6.2). Show relations 23 and 24 for $\mu_{M}$ and $\tilde{\lambda}_{M}$. For all $x, y \in H(A)$ and $m \in H(M)$

$$
\begin{aligned}
& \tilde{\lambda}_{M}\left(\alpha \otimes \tilde{\mu}_{M}\right)(x \otimes y \otimes m)=\tilde{\lambda}_{M}\left(\alpha(x), \tilde{\mu}_{M}(y \otimes m)\right) \\
= & \tilde{\lambda}_{M}\left(\alpha(x), \mu_{M}\left(\alpha^{2} \otimes I d\right)\right)(y \otimes m) \\
= & \lambda_{M}\left(\alpha^{2} \otimes I d\right)\left(\alpha(x), \mu_{M}\left(\alpha^{2}(y) \otimes m\right)\right) \\
= & \lambda_{M}\left(\alpha^{3}, \mu_{M}\left(\alpha^{2} \otimes m\right)\right) \\
= & \mu_{M}\left(\left\{\alpha_{A}^{2}(x), \alpha_{A}^{2}(y)\right\}, \alpha_{M}(m)\right) \\
& +\varepsilon(\mathrm{x}, \mathrm{y}) \mu_{\mathrm{M}}\left(\alpha_{A}^{3}(y), \lambda_{M}\left(\alpha_{A}^{2}(x), m\right)\right)(b y(23)) \\
= & \mu_{M}\left(\alpha^{2}(\{x, y\}), \alpha_{M}(m)\right) \\
& +\varepsilon(\mathrm{x}, \mathrm{y}) \mu_{\mathrm{M}}\left(\alpha^{2} \otimes I d\right)\left(\alpha(y), \lambda_{M}\left(\alpha^{2} \otimes I d\right)(x \otimes m)\right) \\
= & \mu_{M}\left(\alpha^{2} \otimes I d\right)\left(\{x, y\} \otimes \alpha_{M}(m)\right)+\varepsilon(\mathrm{x}, \mathrm{y}) \tilde{\mu}_{M}\left(\alpha(y), \tilde{\lambda}_{M}(x \otimes m)\right) \\
= & \tilde{\mu}_{M}\left(\{x, y\} \otimes \alpha_{M}(m)\right)+\varepsilon(\mathrm{x}, \mathrm{y}) \tilde{\mu}_{M}\left(\alpha(y), \tilde{\lambda}_{M}(x \otimes m)\right) .
\end{aligned}
$$

And, for $x, y \in H(A)$ and $m \in H(M)$

$$
\begin{aligned}
& \lambda_{M}\left(\mu \otimes \alpha_{M}\right)(x \otimes y \otimes m) \\
= & \lambda_{M}\left(\alpha^{2} \otimes I d\right)\left(\mu(x \otimes y) \otimes \alpha_{M}(m)\right) \\
= & \lambda_{M}\left(\alpha^{2}(\mu(x \otimes y)) \otimes \alpha_{M}(m)\right) \\
= & \mu_{M}\left(\alpha^{3}(x), \lambda_{M}\left(\alpha^{2}(y), m\right)\right) \\
& +\varepsilon(\mathrm{x}, \mathrm{y}) \mu_{\mathrm{M}}\left(\alpha^{3}(y), \lambda_{M}\left(\alpha^{2}(x), m\right)\right) \quad(b y(24)) \\
= & \mu_{M}\left(\alpha^{2} \otimes I d\right)\left(\alpha(x), \lambda_{M}\left(\alpha^{2} \otimes I d\right)(y \otimes m)\right) \\
& +\varepsilon(\mathrm{x}, \mathrm{y}) \mu_{\mathrm{M}}\left(\alpha^{2} \otimes I d\right)\left(\alpha(y), \lambda_{M}\left(\alpha^{2} \otimes I d\right)(x \otimes m)\right) \\
= & \tilde{\mu}_{M}\left(\alpha(x), \tilde{\lambda}_{M}(y \otimes m)\right)+\varepsilon(\mathrm{x}, \mathrm{y}) \tilde{\mu}_{M}\left(\alpha(y), \tilde{\lambda}_{M}(x \otimes m)\right) .
\end{aligned}
$$

Hence equations 23 and 24 hold for $\mu_{M}$ and $\tilde{\lambda}_{M}$. This completes the proof.

\section{Corollary 6.3}

Let $(A, \mu,\{.,\},. \varepsilon)$ be $\mathrm{A} \varepsilon$ commutative color Poisson algebra and $\left(M, M_{M}, \lambda_{M}, \alpha_{M p}\right)$ A module over the color Hom-Poisson algebra $\left(A, \mu_{\alpha}=\alpha \circ \mu,\{., .\}_{\alpha}=\alpha \circ\{.,\},. \varepsilon, \alpha\right)$.Then $\tilde{\mu}_{M}, \tilde{\lambda}_{M}$ define another color Hom- Poisson module structure on M.

\section{References}

1. Makhlouf A, Silvestrov S (2010) Notes on Formal Deformations of Homassociative algebras and Hom-Lie algebras. Forum Math 22: 715-739.

2. Yuan L (2012) Hom-Lie color algebras. Communication in algebra40: 575-592.

3. Abdaoui K, Ammar F, Makhlouf A (2013) Constructions and cohomology of color Hom-Lie Algebras.

4. Ammar F, Makhlouf A (2010) A Hom-Lie super algebras and Hom-Lie admissible super algebras. J Algebra 324: 1513-1528.

5. Scheunert M, Zhang RB (1998) Cohomology of Lie super algebras and their generalizations. J Mathematical Physics 39:5024. 
6. Ammar F, Saadaoui N, Makhlouf A (2013) Cohomology of Hom-Lie super algebras and q-deformed Witt super algebra. Czechoslovak Mathematical Journal 63: 721-761.

7. Scheunert M (1979) The theory of Lie super algebras: An introduction. Lecture Notes in Mathematics 716:1-247.

8. Feldvoss J (2001) Representations of Lie colour algebras. Adv Math 157: 95-137.

9. Farkas DR (2000) Modules for Poisson algebras. Communication in algebra 28: 3293-3306.

10. Caressa P (2000) Examples of Poisson- Modules I.RendicontidelCircoloMatem atico di Palermo 3: 419-452.

11. Yau Donald (2010) Non-commutative Hom-Poisson algebras.
12. Pichereau A (2006) Poisson (co)homology and isolated singularities. J Algebra 299: 747-777.

13. Launois S, Richard L (2006) Twisted Poisson duality for some quadratique Poisson algebras.

14. Yau Donald (2008) Module Hom-algebras.

15. Humphreys JE (1972) Introduction to Lie algebra and representation theory. Graduate Texts in Mathematics 9: 1-164

16. Williams AA, Steven HW (1992) Algebra, An Approach via Modules Theory Springer Science and Business Media 526.

17. Berrick AJ, Keating ME (2000) Rings and Modules, An introduction with K-theory in view. Cambridge University Press. 